\title{
Simulating the transport of high-energy solar protons during historic GLE events
}

\author{
C.O.G. Waterfall ${ }^{a, *}$ and S. Dalla ${ }^{a, b}$ \\ ${ }^{a}$ University of Central Lancashire, \\ Preston, $U K$ \\ E-mail: cwaterfall@uclan.ac.uk
}

3D test particle simulations of historic ground level enhancement (GLE) events are performed to investigate the propagation and distribution of solar protons within the heliosphere. The test particle model, which includes drift effects, is also used to explore the influence a heliospheric current sheet (HCS) has on the propagation of protons. Historic GLEs with a source location close to and far from the HCS, as well as poorly and well-connected events are considered. The modelling is performed for high energy (300-1200 MeV) protons to represent the energetic conditions under which these GLEs occur. The derived intensity profiles at 1AU are compared to observations from HEPAD onboard GOES as well as neutron monitor data. We present the results from two of our historic GLE simulations here: GLE 42 and 65. Our results suggest that the HCS plays a significant role in distributing energetic particles throughout the heliosphere. The particle transport along the HCS is most efficient when the injection location is very close $\left(<10^{\circ}\right)$ to it. GLEs with flares that are within $10^{\circ}$ of the HCS all have significantly larger neutron monitor increases than those with flares further away, suggesting that the proximity of the flare to the HCS strongly affects the severity of the event at Earth.

$37^{\text {th }}$ International Cosmic Ray Conference (ICRC 2021)

July 12th - 23rd, 2021

Online - Berlin, Germany

\footnotetext{
${ }^{*}$ Presenter
} 


\section{Introduction}

Protons accelerated to relativistic energies in solar eruptive events can be detected by groundbased neutron monitors on Earth. These high energy events are known as ground level enhancements (GLEs). GLEs occur infrequently, with only 72 recorded since the 1940s [1]. However, the energetic nature of these events pose a large risk to humans and infrastructure in space and at high altitudes. With the growing number of satellites and advances in space exploration it is therefore critical to understand more about these GLE events and improve models that forecast them [2].

This proceedings details the results from the modelling of some of these GLE events. Each GLE is simulated with a 3D test particle model that incorporates a heliospheric current sheet (HCS) based on the magnetic field configuration at the time of the event. The role of the HCS in the transport of relativistic protons in the largest GLE events is a focus of this research. The HCS has previously been suggested to influence this heliospheric particle propagation during GLE events, e.g. [3], [4]. The model and its parameters are described in Section 2, followed by the main results of the study and our conclusions in Sections 3 and 4.

\section{Simulations}

The historic GLEs are modelled using a 3D test particle code previously used in simulations of energetic solar particles ([3], [5]). The test particle code includes drifts due to curvature and gradient of the Parker spiral as well as drifts along the HCS. The interplanetary magnetic field (IMF) is modelled as two separate hemispheres of oppositely directed magnetic fields separated by a wavy HCS. The relative direction of the magnetic field lines in each hemisphere is described with either an A+ or A- notation from galactic cosmic ray (GCR) literature. When the field lines point inwards in the northern hemisphere (and out in the south) the polarity configuration is A+. The opposite case is true for an A-configuration. The polarity at the time of each modelled GLE is important as it has been seen to affect drift directions in previous test particle models ([6], [7]). For an A+ configuration, there is significant drift towards and along the HCS towards the West. However, for an A- configuration there is drift away from the HCS towards the poles. If the injection location is close to the HCS there is some drift Eastward along it.

For each simulated event, $3 \times 10^{6}$ protons with energies between $300<\mathrm{E}<1200 \mathrm{MeV}$ are instaneously injected at a height of $1 \mathrm{R}_{\odot}$ above the surface of the Sun. These protons were injected according to a single power law with an index of -1.5 , similar to the power law indices derived from SOHO/EPHIN observations of SEP events [8]. The protons are injected over a source width of $10 \times 10^{\circ}$. The true size of injection regions for GLE events is currently unknown and could be greater than $10^{\circ}$. We have varied this source region up to $60^{\circ}$ to account for this uncertainty, however only the results for a $10^{\circ}$ region is shown in this proceedings as it illustrates the propagation and distribution of the particles more clearly (see Section 3). However, there have been suggestions that GLEs originate from more compact solar regions than for SEP events [9]. The solar wind speed is individually set for each event corresponding to measurements taken by the CELIAS/MTOF Proton Monitor on-board the SOHO Spacecraft. Where solar wind data is unavailable, the speed is set at $500 \mathrm{~km} / \mathrm{s}$. The mean free path is set at $0.3 \mathrm{AU}$ for all our simulations, this is in agreement with similar values obtained from models of past GLE events ([10], [11]). The protons are injected at 
the flare location associated with each individual GLE event. The total simulation time is 72 hours for all events.

\section{Results}

We have modelled over 15 historic GLE events between 1978 and 2017. The GLEs are chosen to cover a range of HCS configurations and different A- or A+ configurations. This time range for GLE events also allows us to compare our results with more sophisticated neutron monitor data as well as GOES HEPAD observations. We have excluded any GLE event from our modelling where the flare and Earth's footpoint have a longitudinal separation of less than $20^{\circ}$. This allows us to see the extent to which the heliospheric current sheet aids in transporting energetic particles further distances towards an observer. We have also separated our events into two categories; those with a flare located within $10^{\circ}$ of the HCS, and those where the flare is $>10^{\circ}$ from the HCS. The following subsections shows the test particle model results for two GLE events (42 and 65) that fall into either category. For each event, the simulation parameters are those described in Section 2 unless otherwise stated.

\subsection{GLE 42: 29th September 1989}

GLE 42, on the 29th September 1989, was one of the most intense GLE events recorded. It had a neutron monitor percentage increase of nearly $400 \%$ [12]. The source surface map (SSM) for this event is shown in Figure 1. This source surface map is obtained from the Wilcox Solar Observatory (WSO) at: http: //wso . stanford . edu. The SSMs are produced from potential field modelling using photospheric magnetogram data, with a source surface radius of $2.5 \mathrm{R}_{\odot}$. Figure 1 shows the SSM for Carrington rotation 1820, covering the GLE 42 time period. The darker regions correspond to inward pointing magnetic field lines, with outwardly pointing field lines represented by the lighter regions. These two regions are separated by a solid white neutral line representing the current sheet. While the current sheet can be flat or wavy in nature, it is often highly disturbed around solar maximum. This is the case in Figure 1. The red lines in Figure 1 are an approximate fit to this distorted current sheet which is used in the test particle simulations.

From Figure 1 it is clear this event falls into the first category, as the flare location (star symbol) is within $5^{\circ}$ of the HCS. The Earth's footpoint (square symbol) is also almost directly on the HCS. Also of note is the orientation of the magnetic field lines. They are mostly pointing inwards in the northern hemisphere, producing an A- IMF configuration. From Figure 1, we therefore expect the particles to mostly drift along the HCS towards the East, in the direction of the Earth's footpoint.

Figure 2 shows the test particle simulation results for GLE 42. This map plots shows the cumulative particle crossings at $1 \mathrm{AU}$ over 72 hours across the entire longitudinal range. The darker red region located at $[90,-26]$ shows the source region where there are a higher number of particle crossings. The contours outwards from this region show decreasing levels of particle counts, with the outer regions of the heliosphere receiving the least particles. The shape of the HCS, illustrated as the red line fit in Figure 1, is outlined by the particle crossings in this Figure 2 map plot. As expected, the Earth (red triangle) is directly in the path of the energetic particles, despite being longitudinally separated from the injection region by more than $30^{\circ}$. 


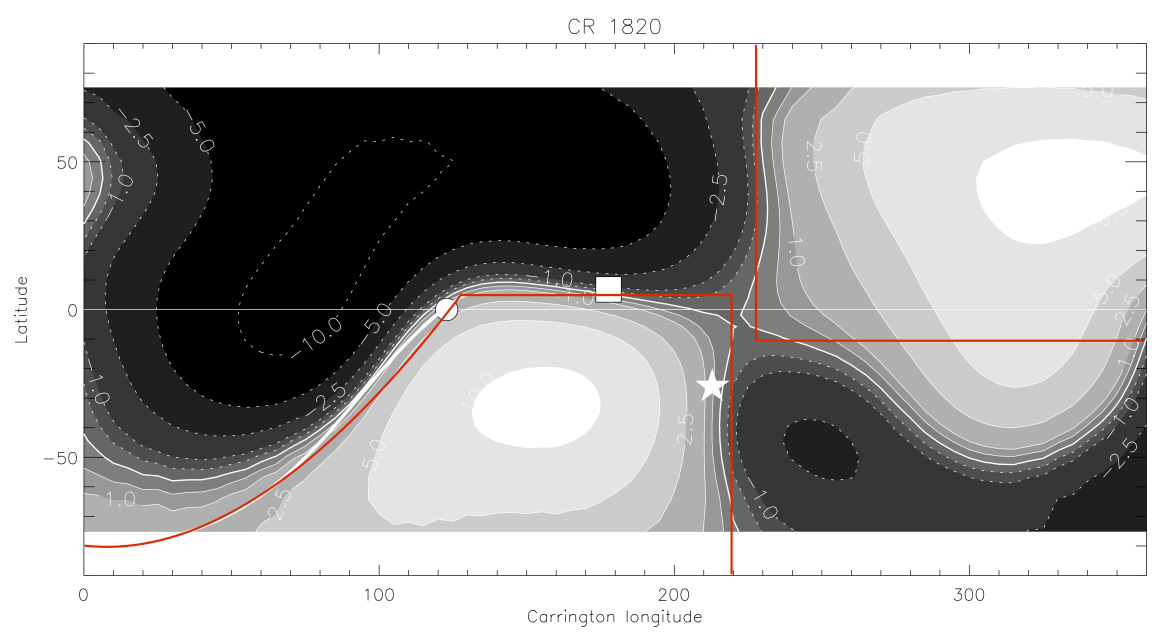

Figure 1: Source surface map for Carrington rotation 1820. The flare and Earth's relative footpoint for GLE 42 are illustrated as the star and square symbol respectively. The red lines are the fit to the heliospheric current sheet described by the SSM's neutral line (solid white line). The circle is the position of the central meridian at the time of the flare.

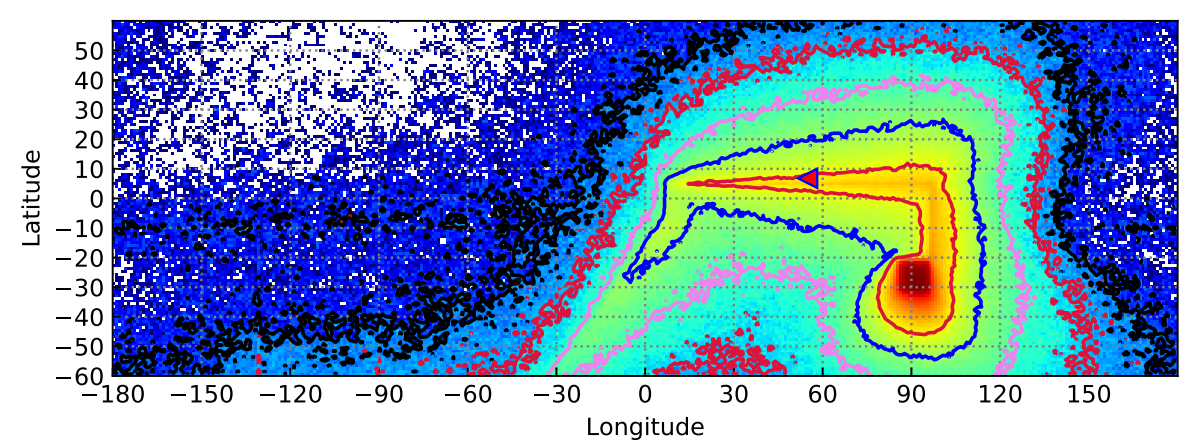

Figure 2: Map plot of cumulative energetic proton crossings at 1AU for the GLE 42 simulation. The total simulation period is 72 hours. The injection region is approximated as the flare location around [90,-26] with the Earth's approximate location at the time of the flare shown as the red triangle. The contours illustrate the number of particle crossings, with more particle crossings focused around the source region and nearby current sheet.

The simulated intensity profile at Earth's footpoint is shown in Figure 3 (green) compared to the observed GOES HEPAD profile (red). Both profiles are shown for the same energy range corresponding to HEPAD's P8 channel for high energy protons. Both profiles are shown for 72 hours, with the HEPAD profile starting from flare onset. The left y-axis gives the HEPAD flux values measured in $\left[\mathrm{cm}^{-2} \mathrm{~s}^{-1} \mathrm{sr}^{-1} \mathrm{MeV}^{-1}\right.$ ], while the right $\mathrm{y}$-axis gives the model intensity in [counts $\mathrm{s}^{-1}$. The simulated and observed profile are similar, with comparable peak fluxes and decay times. Despite the location of the flare being on the limb and therefore not in a region of good magnetic connection (i.e. W40-W60), both intensity profiles have a sharp rise and large peak flux for a high energy channel. The P9 and P10 HEPAD and simulated profiles have a similar profile shape, but with lower peak fluxes (not shown here). The width of the injection region was also increased to 


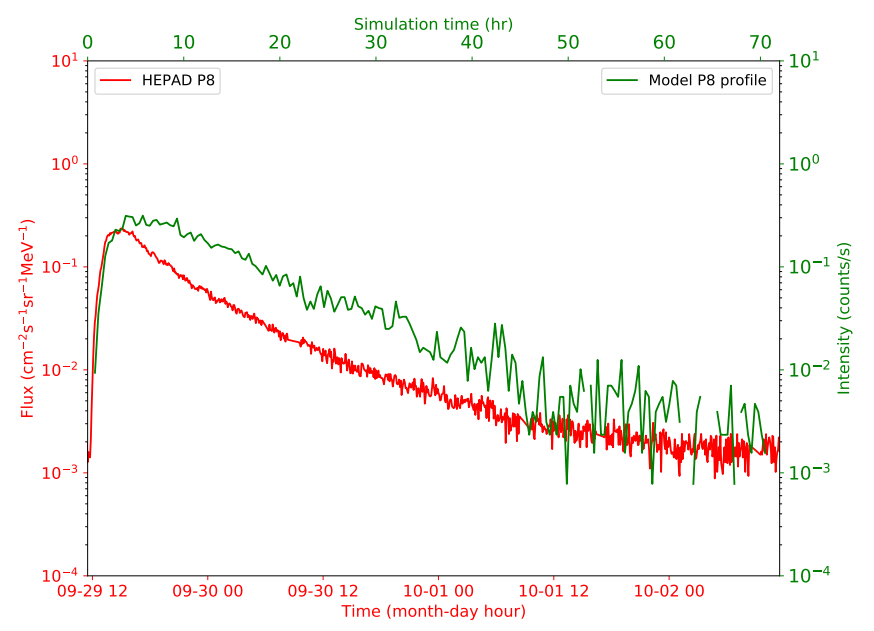

Figure 3: Simulated intensity profile at Earth's footpoint for the GLE 42 simulation (green) and the observed GLE 42 intensity profile for GOES HEPAD (red). Both profiles are shown for the P8 energy channel (350-420 $\mathrm{MeV}$ ) and cover approximately 72 hours each.

$60^{\circ}$ to see the effect on the intensity profile (not shown). Overall the change was not significant, with the $10^{\circ}$ profile fitting the observations better.

\subsection{GLE 65: 28 October 2003}

GLE 65, on the 28th October 2003, is presented here as an example of a GLE where neither the flare or Earth was located close to the HCS. Looking at the SSM for Carrington rotation 2009 in Figure 4 we can see the flare is more than $30^{\circ}$ away from the HCS. The IMF is also in an Aconfiguration, like for GLE 42, however this time the majority of the particle drift will be away from the HCS due to the large separation between the flare and current sheet. It is only when the flare is within $10-15^{\circ}$ of the HCS in an A- configuration that there is significant drift along it.

This drift away from the HCS is illustrated in the map plot for the GLE 65 simulation in Figure 5. The majority of the drift here is towards the poles, with the outline of the HCS barely highlighted by the minimal particle crossings along it. It is evident, when comparing this map plot to that in Figure 2, the difference a flare close to the HCS makes. The distribution and propagation of energetic particles along the HCS is far more likely the closer the source region is to it. However, even with minimal transport along the HCS, particle crossings in Earth's vicinity is still possible depending on the relative position of the Earth to the source region. In Figure 5, the Earth's footpoint is again shown as the red triangle. Despite not being on the current sheet, the Earth is situated in the direction of drift from the source region, i.e. North and more West of the flare. As these simulation include the effects of corotation causing the slight drift to the West in all simulations, the Earth is ideally placed in this scenario.

The corresponding intensity profile at Earth's footpoint for both the test particle simulation and observed by HEPAD is shown in Figure 6. Both profiles are plotted for approximately 72 hours (for the red HEPAD curve the start time is taken as the flare onset time, whereas for the green model curve the start time is taken from the moment the particles are instantaneously injected). GLE 66 is 


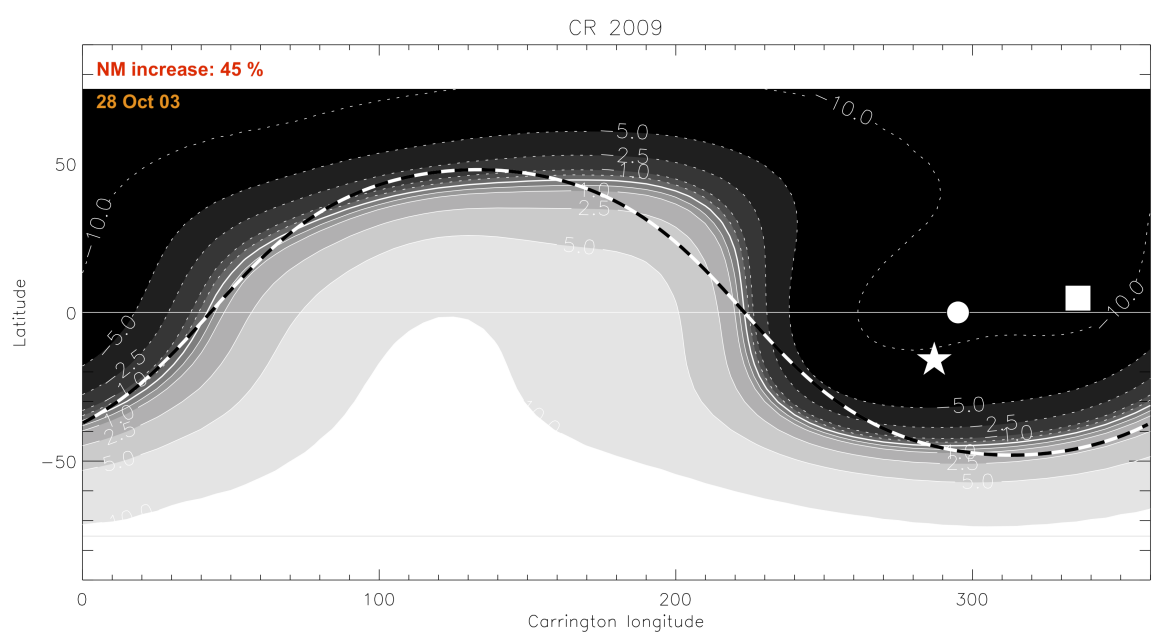

Figure 4: Source surface map for Carrington rotation 2009. The flare and Earth's footpoint for GLE 65 are illustrated as the star and square symbol respectively. The dashed line is the fit to the heliospheric current sheet illustrated by the SSM's neutral line (solid white line).

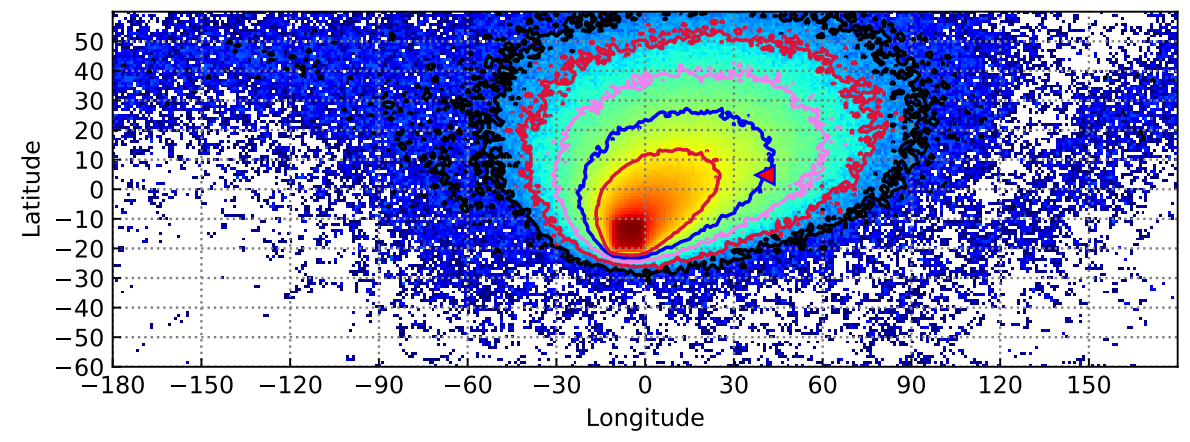

Figure 5: Map plot of cumulative energetic proton crossings at $1 \mathrm{AU}$ for GLE 65 over 72 hours. The protons are injected at the flare location $[-8,-16]$ with the Earth's approximate location at the time of the flare shown as the red triangle. The contours illustrate the number of particle crossings.

visible shortly after GLE 65 in the HEPAD profile but is not modelled here as the test particle code currently only includes single particle injections. The profile shape for GLE 65 is very different to that of GLE 42, with a shallower rise and more gradual profile. The peak flux is also approximately an order of magnitude smaller. The shape of both the model and observed intensity profiles are similar in nature, however there is a delay in the onset of the model profile compared with the observed one. When the Earth's footpoint is in the path of the HCS drift there is no delay (as for GLE 42), suggesting that the propagation along the HCS is a far more efficient means of transport in these simulations than the A- induced drift towards the poles. Removing the HCS from the GLE 65 simulation has no effect on the model intensity profile at Earth. However, removing the HCS from the GLE 42 simulation leads to no particle counts at Earth's location. The drift pattern is similar to that of Figure 5, i.e. towards the North and away from Earth's footpoint. This illustrates the necessity of the HCS in models where the flare is close to it.

As is expected from the lower peak HEPAD flux of GLE 65, this event also had a smaller 


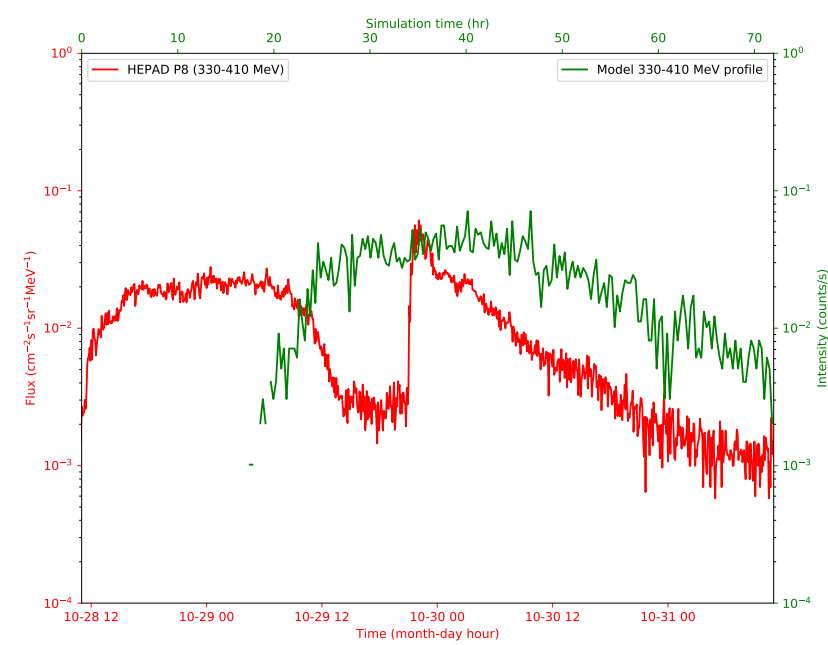

Figure 6: Model intensity profile at Earth's footpoint for GLE 65 simulation (green) and the observed GLE 65 intensity for GOES HEPAD (red). Both profiles are shown for the P8 energy channel (350-420 MeV) and cover 72 hours from flare onset (red) and particle injection (green).

neutron monitor increase of $45 \%$ (compared with $395 \%$ of GLE 42). Neither event was in a particularly favourable location with respect to the magnetic connection between the Sun and Earth. Indeed, the source longitude of $90^{\circ}$ West used for GLE 42 is sometimes quoted as being closer to W100 in literature ([13], [14]). GLE 65 was by no means a small event either, with a flare class of X17 and CME velocity of $2459 \mathrm{~km} / \mathrm{s}$. Comparatively, the flare class for GLE 42 was X9.0. However, GLE 42 was a far larger event at Earth and our results suggest it is the proximity of the flare to the HCS that influences the magnitude of these events at Earth. Indeed, the largest NM events since the 1970s all had source locations within approximately $10^{\circ}$ of the HCS. The largest GLE event on record, GLE 69 on 20 January 2005, had a neutron monitor increase of over $5000 \%$ with both the flare and Earth situated $<5^{\circ}$ from the HCS. Additionally, of the 6 GLEs that have occurred behind the limb since 1975 , all have had a flare located within $15^{\circ}$ of the current sheet.

\section{Conclusions}

These proceedings describe the results obtained from 3D test particle modelling of a selection of historic GLE events. The propagation and distribution of high energy protons within the heliosphere is studied, both with and without a heliospheric current sheet. A variety of events are chosen to ensure a range of HCS configurations chosen, e.g. a flare located both close to and far from the HCS, etc.

These simulations suggest that the HCS plays a significant role in distributing energetic particles throughout the heliosphere. Additionally, this particle transport along the HCS is most efficient when the flare is located within approximately $10^{\circ}$ of the HCS. Included in our selection of events, is the simulation of the 8 largest GLEs since the 1960s. In 7 out of 8 cases the flare is within this $10^{\circ}$ limit of the current sheet, suggesting the proximity of the flare influences the size of the GLE 
at Earth. Also, out of the 6 GLEs with flares occurring behind the limb since the 1970s, all of them had flares within $15^{\circ}$ of the HCS. These proceedings use GLE 42 (and 65) as examples of cases where the flare is close to (and far from) the HCS to illustrate how a proximity to the HCS causes larger peak fluxes and greater particle distribution longitudinally.

In total, we have found the HCS affects our results in $75 \%$ of modelled events. Our simulations show that for events like GLE 65, which do not rely on the HCS to transport particles towards Earth, the relative position between the Earth and flare (rather than flare and HCS) is more important. These results, as well as the simulated intensity profiles, can be used to inform future forecasting models as well as for comparison with existing observations from multiple observers between the source and $1 \mathrm{AU}$.

\section{References}

[1] I. Usoskin, S. Koldobskiy, G.A. Kovaltsov, A. Gil, I. Usoskina, T. Willamo, and A. Ibragimov. $A \& A, 640: \mathrm{A} 17,2020$.

[2] R. Schwenn. Living Reviews in Solar Physics, 3(1):1-72, 2006.

[3] M. Battarbee, J. Guo, S. Dalla, R. Wimmer-Schweingruber, B. Swalwell, and D.J. Lawrence. $A \& A, 612: \mathrm{A} 116,2018$.

[4] C.R.A. Augusto, C.E. Navia, M.N. de Oliveira, A.A. Nepomuceno, A.C. Fauth, V. Kopenkin, and T. Sinzi. Publications of the Astronomical Society of the Pacific, 131(996):024401, 2018.

[5] S. Dalla and P.K. Browning. A \& A, 436(3):1103-1111, 2005.

[6] M. Battarbee, S. Dalla, and M.S. Marsh. The Astrophysical Journal, 854(1):23, 2018.

[7] S. Dalla, G.A. De Nolfo, A. Bruno, J. Giacalone, T. Laitinen, S. Thomas, M. Battarbee, and M.S. Marsh. $A \& \&$, 639:A105, 2020.

[8] P. Kühl, N. Dresing, B. Heber, and A. Klassen. Solar Physics, 292(1):1-13, 2017.

[9] N.V. Nitta, Y. Liu, M.L. DeRosa, and R.W. Nightingale. Space science reviews, 171(1-4):6183, 2012.

[10] J.W. Bieber, W. Droege, P.A. Evenson, R. Pyle, D. Ruffolo, U. Pinsook, P. Tooprakai, M. Rujiwarodom, T. Khumlumlert, and S. Krucker. The Astrophysical Journal, 567(1):622, 2002.

[11] A. Sáiz, D. Ruffolo, M. Rujiwarodom, J.W. Bieber, J. Clem, P. Evenson, R. Pyle, M.L. Duldig, and J.E. Humble. In (ICRC29), Volume 1, volume 1, page 229, 2005.

[12] K.G. McCracken, H. Moraal, and M.A. Shea. The Astrophysical Journal, 761(2):101, 2012.

[13] L.I. Miroshnichenko, C.A. De Koning, and R. Perez-Enriquez. Space Science Reviews, 91(3):615-715, 2000.

[14] H. Moraal and R.A. Caballero-Lopez. The Astrophysical Journal, 790(2):154, 2014. 